\title{
ZWPS and Pressure Scroll: Two Pressure-based Techniques in Pen-based Interfaces
}

\author{
JiBIN YIN ${ }^{\dagger}$ and XIANGSHI REN ${ }^{\dagger}$
}

\begin{abstract}
This paper investigates the interaction ability when introducing pressure into current basic interaction techniques by developing two novel techniques. A Zoom-based technique with pressure (hereafter referred to as ZWPS) is proposed to improve pixel-target selection. In this technique the pressure is used as a switch mode to couple a standard Point Cursor and a zoomable technique together. Pressure Scroll is also presented with a view to advancing scrolling performances by employing arc or line strokes to scroll documents. In this technique pressure is used as an additional control factor to widen the adjustable range of the scrolling velocity. We conducted two experiments to examine the effectiveness of ZWPS and Pressure Scroll. The experimental results indicate that they both bring significant benefits to the users.
\end{abstract}

\section{Introduction}

In traditional GUIs many computing performances such as selection tasks and scrolling tasks are commonly driven by coordinates ( $\mathrm{x}$, y) input from pointing devices (e.g., Mice), binary buttons or Wheels. Some pen-based input devices provide additional inputs such as pressure, which can obviously widen the input bandwidth. Some fundamental studies have reported the benefits of pressure-based interaction as an alternative input channel ${ }^{5), 8), 18)}$. However, with few exceptions ${ }^{17), 18), 20)}$ pressure is seldom coupled with current interaction techniques. Pressure can be typically used to perform interactions by being mapped to several discrete states, or by controlling a continuous variable. The investigation of the potentials of these two ways of using pressure for interaction techniques should be performed. Therefore, this study seeks to look at two novel techniques which incorporate pressure to enhance user performance and preference.

First, we propose a hybrid selection technique comprising a standard Point Cursor and a zoomable technique, using pressure as the switch mode, to enable a quick pixel-level target selection. ZWPS employs the discrete state of pressure to widen the performance of target selection. It has two major advantages: first, it zooms only the specified area to facilitate pixel-level targets, without breaking overview information in interfaces; second, it maintains standard pointing manners for selecting normal or big targets. We also propose a new

$\dagger$ Kochi University of Technology, Japan scrolling technique, Pressure Scroll, which we developed to facilitate scrolling tasks in penbased interfaces. Pressure Scroll uses the continuous variable of pressure to enhance scrolling performance and utilizes strokes (circular or linear) to control scrolling velocity and variable pressure is utilized to further finely adjust the scrolling velocity.

In the following sections, we will review related work; discuss the design and implementation of ZWPS and Pressure Scroll; evaluate the performance of these two techniques in two experiments; and conclude by discussing implications for user interface design.

\section{Related Work}

The related work to this study includes pen pressure researches, precise selection techniques and scrolling techniques.

\subsection{Related Work on Pressure}

Studies on pressure can be roughly divided into two categories. One category investigates the general capabilities of humans to interact with computers using pressure. For example, Herot and Weinzapfel ${ }^{8)}$ investigated the human ability of the finger to apply pressure and torque to a computer screen. Buxton ${ }^{5)}$ studied the use of touch-sensitive technologies and the possibilities for interaction they suggest. Ramos, et al. ${ }^{18)}$ explored the human ability to vary pen-tip pressure as an additional channel of control information.

The other category of study is where researchers build pressure-enabled applications or techniques. For instance, Ramos and Balakrishnan ${ }^{17)}$ demonstrated a system called LEAN and a set of novel interaction techniques for 
the fluid navigation, segmentation and annotation of digital video. Ramos and Balakrishnan ${ }^{20)}$ designed the Zlider widget that used pressure to produce different scales to achieve high precision parameter manipulation. $\mathrm{Li}$, et al. ${ }^{13)}$ investigated the use of pressure as a possible means to delimitate the input phases in pen-based interactions. Although these works opened the door to establish pressure as a research avenue, we are unaware of any work which addressed the issue of applying pressure in selection or scrolling techniques. Thus, we attempt to investigate these potentials in this paper.

\subsection{Related Work on Precise Selection Techniques}

On many occasions there are requirements for precise selection such as searching for a location in a map application. This issue was notably addressed by Sears, Shneiderman and their colleagues ${ }^{16), 22), 23)}$. Their basic technique, called Take Off, provides a cursor above a pen-tip or the user's finger tip with a fixed offset when touching the screen to achieve precise selection. Zoom pointing is also a typical technique for small target selection, which is currently used in many painting systems. Ren and Moriya ${ }^{21)}$ investigated different strategies for handling small targets and reported that $1.8 \mathrm{~mm}$ ( 5 pixels) was a crucial limit beyond which special needs arise. Worden, et al. ${ }^{26)}$ proposed an enhanced Area Cursor to alleviate the ambiguity, by including a single point hotspot centered within the area cursor, which took effect when more than one target was within the cursor's bounds. The enhanced Area Cursor performed identically to regular point cursors when targets were close together, and it outperformed point cursors when targets were far apart. Ramos, et al. ${ }^{19)}$ presented pointing lenses techniques to help users easily select targets by offering them an enlarged visual and interaction area. Experimental results showed that Pressure-Activated Lens was the top overall method and all activation lenses offered benefits for selecting targets of less than 5 pixels. Albinsson and Zhai ${ }^{2)}$ proposed PrecisionHandle and Cross-Keys to complement existing techniques for touch screen interaction. We present ZWPS which integrates two techniques (Point Cursor and the zoomable technique) by using pressure as the switch mode to allow both

\footnotetext{
is The standard regular cursor in GUIs.
}

precise and imprecise selections.

\subsection{Related Work on Scrolling Tech- niques}

Scrolling is also a fundamental task, particularly for devices with small screens. Igarashi and Hinckley ${ }^{10)}$ proposed a technique called SDAZ (speed dependent automatic zooming). When using it to scroll documents, the documents are automatically zoomed-out as the scroll rate increases. By automatically zooming, the visual flow rate is reduced enabling rapid scrolling without motion blur.

There are efforts that explore navigation techniques in mobile devices with small screens. Baudisch, et al. ${ }^{3)}$ proposed a Collapse-toZoom technique which exploits strokes to delete the unimportant contents and highlight the selected ones to enhance navigation performances. MacKay, et al. ${ }^{14)}$ presented a field study comparing software-based navigation techniques (Scroll Bar, tap-and drag, and touch-n-go) on mobile devices. They explored the efficiency and user preferences of these navigation techniques for different levels of mobility (sitting, walking and standing). The works that are more related to ours are those of Smith, et al. ${ }^{24)}$ and Moscovich, et al ${ }^{15)}$. Smith, et al. presented a radial scrolling tool, which exploits circular gestures on the interface to scroll documents. The document can be advanced or reversed by clockwise or counterclockwise circular stroke gestures. Moscovich, et al. presented a virtual scroll ring which simulates the hardware scroll ring device that maps a circular finger motion into a vertical scrolling, to navigate the document. Both of these utilize arc strokes to perform scrolling tasks. Our Pressure Scroll introduces pressure as an additional scale trigger to advance scrolling performance, which is simultaneously controlled by strokes (both arc and line).

\section{Pressure-based Techniques}

Pessure is a continuous scalar value acquired from the interaction of pens and screens. Generally, during a computer interaction a desired object can be controlled by two basic operation modes: using a continuous value to adjust its attributes (e.g., shape, position or color lightness) and using discrete values to change its states (e.g., highlight or not). Correspondingly, there are respectively two fundamental control manners to map pressure to the above operation modes: mapping pressure into a con- 
secutive value, or mapping pressure into discrete states during a performance. Based on the demonstration above this study explores the potentials of incorporating pressure to computing interactions by proposing two novel techniques: ZWPS and Pressure Scroll. The following sections describe these two techniques in detail.

\subsection{Zoom-based Selection Technique with Pressure (ZWPS) for Pixel- level Targets in Pen-based Inter- faces}

Pen-based devices (e.g., Tablet PCs) can be directly manipulated by a pen, even by a bare finger. When targets are very small, especially at pixel-level, such target selections become very difficult for a pen or a finger to perform. In current applications (e.g., Photoshop) such problems are resolved by zooming. For example, drawing a precise line from one dot (e.g., railway station) to another (e.g., a college) on a digital map requires the user to zoom in and click on one end, zoom out to find the other end, zoom in again to click on the other end, then zoom out again to look at both ends, all of which cause great inconvenience to users. Moreover, after zooming in, the user may lose information in the overview and the detailed windows. The switch between zooming in and zooming out often disrupts the user's attention. This awkward situation becomes our direct motivation to study alternatives to the current technique.

We seek to find a selection technique that satisfies the following conditions:

- Have a zoomable function to allow a pixel target selection.

- Provide a flexible and seamless switch mode between zooming in and out.

- zoom only a certain part of the display area to prevent the user from losing the information in overview.

Therefore, we present a novel technique called ZWPS to enhance pixel-target selection. ZWPS, in essence, is a hybrid selection technique that includes two selection techniques: the standard Point Cursor and the zoomable technique. The standard pointing selection mode works in selecting common size targets while zoom selection mode works best when selecting small size targets.

To couple the two techniques in ZWPS a suitable switch mode is essential. The properties of pressure (easily produces controlled discrete

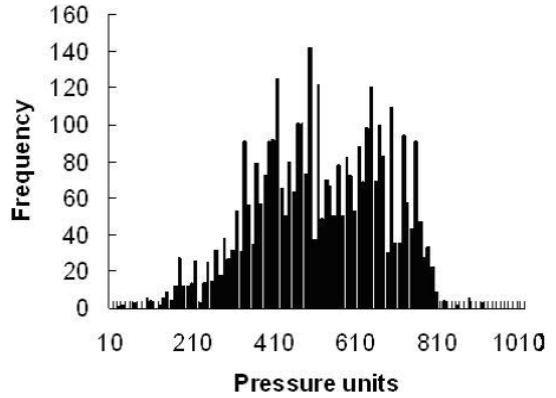

Fig. 1 Pressure distribution histograms for three tasks combined.

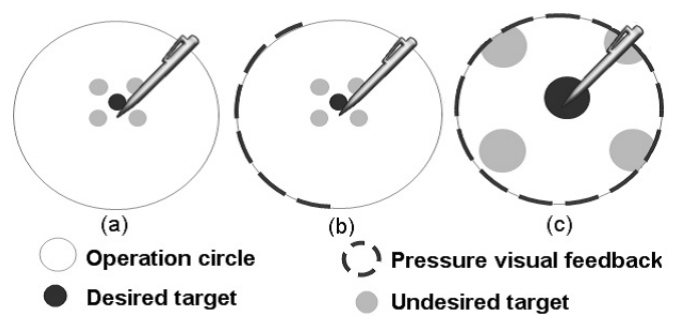

Fig. 2 (a) the operation circle appears when the pen tip is landed on the screen. (b) a dotted arc is used to show pressure amount as visual feedback. (c) the zoomable function is activated by pressure that surpasses a specified threshold.

states) make it appropriate to seamlessly switch modes in ZWPS. To determine a proper switch threshold, a pilot study with 10 subjects was performed. The task was to draw freehand strokes (arbitrary curves and straight lines), basic geometrical graphs (such as rectangles and circles) and a mixed set of Roman, Japanese, and Chinese (kanji) characters and signatures on a blank space in a natural manner. Pentip pressure was recorded in a $17 \mathrm{~ms}$ sampling periods. Ninety-five percent of the force samples fell within the 210 to 810 units range (see Fig. 1). The results showed that pressure levels of more than 810 units were seldom used in a natural manner. Therefore, in ZWPS the threshold value is set at 970 units.

An operation circle (see Fig. 2 (a)) is also defined to specify a zoomed area. When a user lands a pen tip on a screen surface an operation circle appears. The pen tip is always at the center of the operation circle. When the user slides the pen tip on the screen surface the operation circle follows it. Contents that are enclosed by the circle can be zoomed in by imposing heavy pressure, enough to surpass the switch threshold. The diameter of the operational circle was set at $24 \mathrm{~mm}$ by pilot studies. An arc with a dotted red line, which is attached 
on the border of the operational circle, is employed as visual feedback to indicate the current pressure state. When the arc, augmented by pressure from a pen, increases to a complete circle (surpassing the threshold) it means that the zoom technique is activated, Conversely, ZWPS maintains the standard Point Cursor when effective pressure is not applied. When more than one target is enclosed by the operation circle, they are zoomed in or out together simultaneously. The parts of the zoomed contents that are beyond the border of the operation circle cannot be shown in the operation circle (see Fig. $2(\mathrm{c})$ ). If the desired target is not included in the operation circle the user can move the circle to include it in the operation circle by sliding the pen tip. In our current implementations we used a zoom factor of 4 . A very small zoom factor value cannot offer significant benefits for small target selections, while a big value will enable little content to be shown in the operation circle. We found through early pilot studies that a value of 4 resulted in an acceptable performance.

When selecting a target of normal or big size, the user, imposing light pressure on a pen-tip, taps the target directly to select it in the standard selection mode. When selecting a very small target, the target is first enclosed (Fig. 2 (a) and 2 (b)) by the operational circle, and then the zoom selection mode is activated by heavy pressure (surpassing the specified threshold) to enlarge the target. When the user slides the pen tip into the desired target it is highlighted (preselected) (Fig. 2(c)). When the pen tip is lifted from the screen, the target is selected and it recovers its original size. Note that ZWPS only zooms in the operation circle area around the cursor, not the whole area in the view window, which enables the user to avoid losing the information in overview, even when it is in zoomed status.

\subsection{Pressure Scroll}

Scrolling tasks are also very common operations. A scrolling technique that possesses speed and accuracy (i.e., quickly finds the object in a document and accurately relocates it to a desired position) is a good one. In traditional GUIs Scroll Bar is a classic widget which is used to navigate a document. In pen-based systems, Scroll Bar is still used even though some of its characteristics are not suitable for pens. Some researchers propose some new scrolling techniques that use a circular

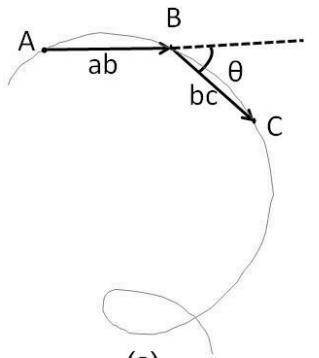

(a)

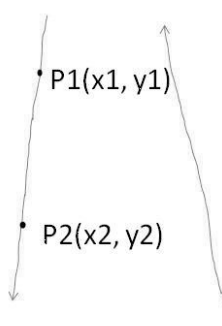

(b)
Fig. 3 (a) Arc: to scroll a document using an arc stroke; (b) Line: to scroll a document using a line stroke.

stroke to achieve a good scrolling performance for pen-based systems ${ }^{15), 24)}$. We also found that a line stroke is good to fluently perform scrolling tasks. In Pressure Scroll we explore the possibility of integrating pressure with the stroke-based scrolling technique. Pressure is utilized as an additional control factor to widen the adjustable range of scrolling velocity (e.g., generating a very fine or a very large scale).

We first illustrate how to achieve scrolling performance only by arc and line strokes. In our implementations, when an arc stroke is drawn to drive scrolling tasks, the coordinates of all points contained in the strokes are recorded to determine the direction and the displacement of the scrolled document. A minimum of three points, $A, B$, and $C$, are required (as shown in Fig. $3(\mathrm{a})$ ). Two vectors indicate the previous and current points passed by the pen. The scrolling direction is determined by the sign of the dot product of the previous vector $\overrightarrow{\mathbf{a b}}$ and the current vector $\overrightarrow{\mathbf{b c}}$. If the sign is positive the direction of rotation is clockwise, and the movement direction is up. The angle difference $\theta$ between the two vectors determines the scrolling displacement.

When a line stroke is drawn to drive scrolling tasks, as shown in Fig. 3 (b), $P_{1}$ and $P_{2}$ indicate the previous and current points passed by the pen. The sign of $\left(y_{2}-y_{1}\right)$ (i.e., direction of the line stroke) determines the scrolling direction of the document: if the direction of the line stroke is up, the document will scroll up, while drawing the stroke in a downwards direction causes the document to scroll down. For vertical scrolling, the vertical difference $\left(\left|y_{2}-y_{1}\right|\right)$ between the previous and current points determines the scrolling displacement; for horizontal scrolling the horizontal difference $\left(\left|x_{2}-x_{1}\right|\right)$ de- 
termines the displacement. Moreover, the line stroke is a much more natural manner and has the advantage of visual consistency (i.e., the direction of the stroke is consistent with the scrolling direction of the document). We can calculate the scrolling velocity produced only by the strokes (hereafter referred to as $\mathbf{V}_{s}$ ) according to the scrolling displacement and the time that is spent to produce this displacement.

In Pressure Scroll, scrolling velocity (i.e., $\mathbf{V}_{\text {scroll }}$ ) is controlled by two factors: strokes and pressure. As shown in Formula 1, $\mathbf{V}_{s}$ is the velocity produced only by the strokes. We use an exponential function to calculate the scale factor by the pressure, where $f(p)$ is a mapping function of the stylus' reported pressure at a particular time.

$$
\mathbf{V}_{\text {scroll }}=\mathbf{V}_{s} \cdot e^{f(p)}
$$

Here, the pressure mapping function $f(p)$ is described. As found by previous work ${ }^{4)}$, the degree of pen pressure perceived by human users is not consistent with that sensed by a digitizer. For example, at a low spectrum of pen pressure, the sensed pressure value increases much faster than users would expect. Previous work ${ }^{4)}$ has used a sigmoid transfer function to account for the effects produced by pressure. In our techniques we also employed the sigmoid transfer function. A number of aspects in the application of pressure have been identified: an initial "dead zone", slow response at low pressure levels (where pressure is too sensitive for users to control) smooth change at median pressure levels (where users have good control of pressure), and quick response at high pressure levels (where force applied by the user can produce tremors, causing sudden pressure variations). We employed a piecewise linear function to approximate the pressure mapping function $f(p)$ (see Eq. $(2))$.

$$
f(p)= \begin{cases}0 & 0 \leq \mathrm{p}<50 \\ (0.33 p-16.67) / 350 & 50 \leq \mathrm{p}<200 \\ (3.14 p-578.57) / 350 & 200 \leq \mathrm{p}<270 \\ (0.39 p+164.53) / 350 & 270 \leq \mathrm{p}<910 \\ (1.59 p-926.9) / 350 & 910 \leq \mathrm{p}<1024\end{cases}
$$

Here, $p$ is the pressure input that ranges from 0 to 1024 .

\section{Experiment 1: ZWPS}

In Experiment 1, to evaluate the performance of ZWPS, we conducted a quantitative experiment to compare it with Take Off and with the improved Area Cursor. We chose Take Off, a promising technique, as the baseline, because

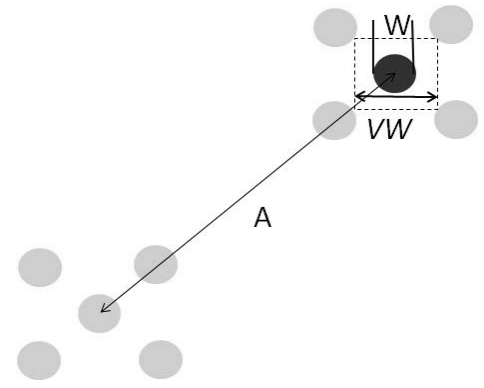

Fig. 4 Experimental setup. The green target in the center around four red circles is the goal target. The four red circles around the goal targets are distracters which determined the $V W / W$ ratio.

it is a very common baseline in many other evaluations of techniques for small-target selection reported to date ${ }^{16), 22), 23)}$. Area Cursor has a larger than normal activation area. This characteristic enables more efficient target acquisitions. However, there are potential problems associated with the implementation of Area Cursor. For example, when more than one target is within the activation hotspot, it is difficult for Area Cursor to perform selection tasks. Therefore, we employed the improved Area Cursor devised by Worden, et al. ${ }^{26)}$. As discussed in Section 2.2, even with the enhancement suggested by Worden, et al. ${ }^{26)}$, Area Cursor did not deliver any benefits when the targets were close together (Area Cursor simply behaved as a point cursor in this situation). We wonder if ZWPS offers some advantages in selecting small targets surrounded with the closed distracter targets. We, therefore, employed a virtual target width $(V W)$ (as shown in Fig. 4) and designed the different $V W / W(2,3$ and 7$)$ ratios as the setting of the experimental design. The virtual target reflects the amount of void space immediately around a target, which, in our experimental settings, is a square and is determined by four distracter targets placed on the four vertexes of the square. In Experiment 1 , the operation circle of ZWPS was set at 81 pixels (about $24 \mathrm{~mm}$ ) and the zoom factor value was set at 4 . The width size of area cursor was 12 pixels, which followed Worden et al.'s design on Area Cursor.

\subsection{Apparatus}

The hardware used in Experiment 1 was a Wacom DTI-520 interactive LCD graphics display tablet with a resolution of $1,024 \times 768$ pixels $(1$ pixel $=0.297 \mathrm{~mm})$, using a wireless stylus that has a pressure sensitive isometric tip (the width of the pen-tip is $1.76 \mathrm{~mm}$ ). It reports 512 

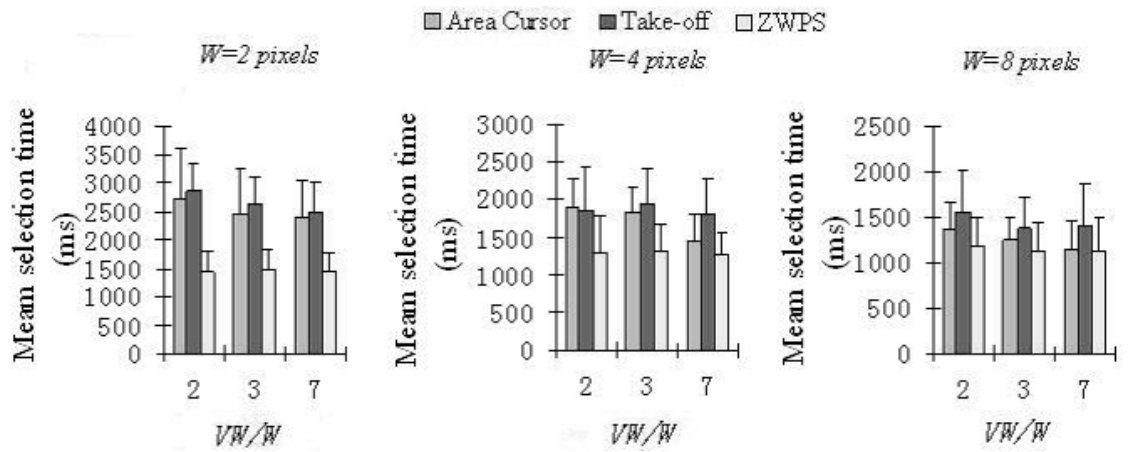

Fig. 5 The mean selection time for the different sizes of targets at each $V W / W$ ratio.

levels (ranging from 0 to 1,024 , the minimum unit is 2) of pressure and has a binary button on its barrel. The experimental software ran on a $3.2 \mathrm{GHz}$ P4 PC with the Windows XP Professional operating system.

\subsection{Participants}

Twelve subjects (10 male and 2 female) all with previous experience using computers were tested for the experiment. The average age was 23.9 years. All subjects used the pen in the right hand.

\subsection{Procedure}

The experimental task was a reciprocal pointing task in which the subjects were required to select two fixed targets back and forth in succession, but, to simulate a more realistic twodimensional pointing environment, we used a multi-directional reciprocal pointing task which included reciprocal diagonal movements. The targets were drawn as solid circles, and were located at various distances from each other along two directional axes. The goal target, the one intended to be selected, was colored green. When a goal target had been selected, it changed color to red which was an indication that the user now had to select the next goal target. Four red circles were placed around each goal target to control the $V W / W$ ratio (see Fig. 4).

The subjects were instructed to select the two goal targets alternately. They were told to emphasize both accuracy and speed. When the subject correctly selected the target, he/she heard a beep sound and the targets swapped colors, which was an indication of a new trial. At the start of each experiment, subjects were given a warm-up block to familiarize themselves with the task and the conditions.

\subsection{Design}

A within-subject design was used. The in- dependent variables were: selection techniques ST (ZWPS, Take Off and the improved Area Cursor), amplitude $A$ (100, 250, 750 pixels), width $W(2,4,8$ pixels), $V W / W$ ratios $(2$, 3,7 ), and direction $D R$ (2 diagonals). A full crossed design resulted in 162 combinations of $S T, A, W, V W / W$, and $D R$. Each combination consisted of 4 selection attempts (i.e., 3 reciprocal movements between the two targets). Each subject performed the experiment in one session lasting approximately forty minutes, depending on each subject's proficiency in selecting the targets. The session was broken up according to the selection techniques.

\subsection{Results}

An ANOVA (analysis of variance) with repeated measures was used to analyze the performance in terms of selection time, error rate, and subjective preference. Post hoc analysis was performed with Tukey's Honestly Significant Difference (HSD) test.

\subsubsection{Selection Time}

There was a significant difference in the mean selection times among the three selection techniques, $\mathrm{F}(2,33)=7.95, \mathrm{p}<.01$. The overall mean selection times were $1,589 \mathrm{~ms}$ for Area Cursor, 1,929 ms for Take Off and 1,288 ms for ZWPS. Tukey HSD tests showed that ZWPS was significantly faster than both Area Cursor and Take Off $(\mathrm{p}<.05)$. No significant difference was found between Area Cursor and Take Off.

As shown in Fig. 5, at the width of 2 there was a significant difference in selection time between the three selection techniques, $\mathrm{F}(2,33)=13.9,11.8$ and 12.9 for the $V W / W$ ratio values of 2,3 and 7 respectively, all $\mathrm{p}<.001$. For each $V W / W$ ratio value Tukey HSD tests showed ZWPS was significantly faster than Area Cursor and Take Off $(\mathrm{p}<.001)$, however, no significant difference was found between 
Area Cursor and Take Off. At the width of 2 , for each $V W / W$ ratio, there was a little void space around the goal target. ZWPS still delivered benefits for such selection tasks by enlarging goal targets, while Area Cursor had no advantages in such situations because it too easily covered more than one target (Area Cursor simply behaves as Point Cursor in this situation) at the same time. Take Off was also worse than ZWPS in selecting very small targets.

At the width of 4 there was a significant difference in selection time between the three selection techniques, $\mathrm{F}(2,33)=5.89,9.41$ and 6.19 for the $V W / W$ ratio values of 2,3 and 7 respectively, all $\mathrm{p}<.001$. For $V W / W$ ratio values of 2 and 3, Tukey HSD tests showed ZWPS was significantly faster than Area Cursor and Take Off $(\mathrm{p}<.05)$, however, no significant difference was found between Area Cursor and Take Off. The results followed the trends similar to selecting small closed targets (at the width of 2). For the $V W / W$ ratio values of 7, Tukey HSD tests showed ZWPS was significantly faster than Take Off $(\mathrm{p}<.01)$, however, there was no other significant difference across selection techniques. Although the targets were still small, the void space around them became bigger. Area Cursor began to show its benefits. ZWPS was comparable to Area Cursor.

At the width of 8 there was no significant difference in selection time between the three selection techniques for each $V W / W$ ratio value. When the targets and their surrounding void space both became bigger, the three techniques were comparable.

Fitts' law ${ }^{7)}$ is commonly used to predict the time it takes to move a mouse pointer from one location to another.

$$
M T=a+b \log _{2}\left(\frac{A}{W}+1\right)
$$

According to Fitts' law, the cursor movement time $(M T)$ increases linearly with the Index of Difficulty $(I D)$, which relies on the logarithm of the distance moved (the amplitude, $A$ ) and the width of the target $(W)$. The two constants, $a$ and $b$, are determined empirically.

We applied Fitts' law to evaluate the experimental results. For ZWPS and Area Cursor we used the effective width (e.g., the effective width $=$ the zoomed width for ZWPS) instead of the physical width. As seen in Fig. 6 ZWPS was modeled by Fitts' law $\left(R^{2}=0.98\right)$, which indicated that the zoomed size of the targets

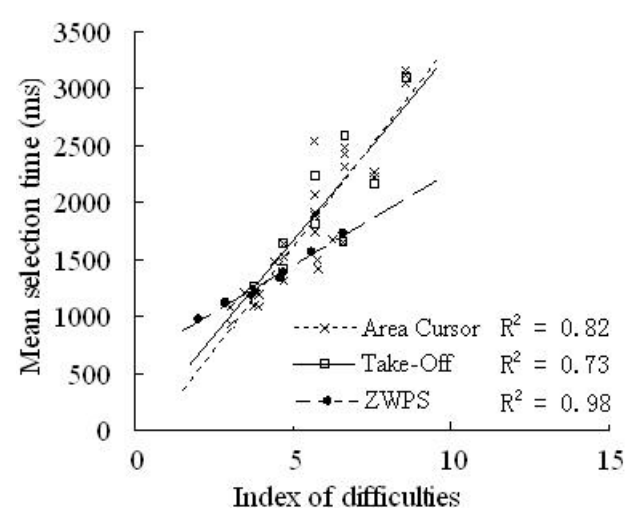

Fig. 6 Line regression of index of difficulty against selection time.

could be utilized fully. Area Cursor and Take Off were only roughly modeled by Fitts' law, $R^{2}$ $=0.82$ for Area Cursor and $R^{2}=0.73$ for Take Off. The relatively low $R^{2}$ for Area Cursor and Take Off may be due to their special selection mechanism and the complex selection tasks in Experiment 1.

\subsubsection{Error Rate}

There was a significant difference in the overall mean error rate between the three techniques, $\mathrm{F}(2,33)=55.7, \mathrm{p}<.0001$. Tukey HSD tests showed ZWPS was significantly more accurate than both Area Cursor and Take Off $(\mathrm{p}<.001)$ in error rate. Take Off was significantly more accurate than Area Cursor $(\mathrm{p}<.001)$. Overall error rates were $31.8 \%$ for Area Cursor, $12.6 \%$ for Take Off, and $5.5 \%$ for ZWPS.

As shown in Fig. 7, at the width of 2 there was a significant difference in error rate between the three selection techniques, $\mathrm{F}(2,33)=40.4$, 63.2 and 45.5 for the $V W / W$ ratio values of 2,3 and 7 respectively, all $\mathrm{p}<.0001$. For each $V W / W$ ratio value, Tukey HSD tests showed ZWPS was significantly more accurate than both Area Cursor and Take Off $(\mathrm{p}<.001)$ in error rate. Take Off was significantly more accurate than Area Cursor $(\mathrm{p}<.001)$.

At the width of 4 there was a significant difference in error rate between the three selection techniques, $\mathrm{F}(2,33)=12.4$ and 15.6 for the $V W / W$ ratio values of 2 and 3 respectively, both $\mathrm{p}<.0001$. No significant difference was found for the $V W / W$ ratio values of 7 . For the $V W / W$ ratio values of 2 and 3 , Tukey HSD tests showed ZWPS and Take Off were both significantly more accurate than Area Cursor $(\mathrm{p}<.001)$ in error rate, however, there was no 

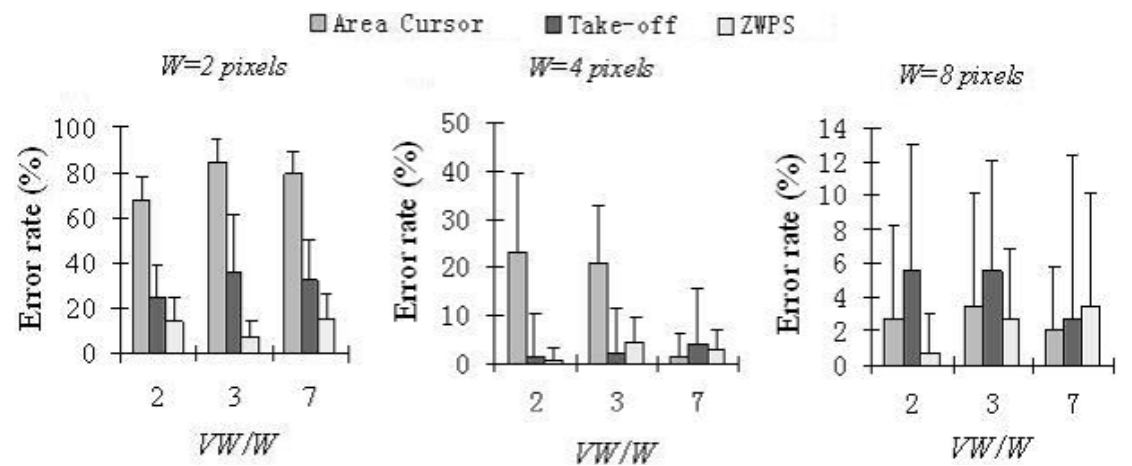

Fig. 7 The error rate for different sizes of targets at each $V W / W$ ratio value.

significant difference between ZWPS and Take Off.

At the width of 8 there was no significant difference in error rate between the three selection techniques for each $V W / W$ ratio value.

From accuracy analysis it was shown that ZWPS significantly outperformed Area Cursor and Take Off for small targets close together, which reflected the effectiveness of its zoomable function.

\subsubsection{Subjective Preference}

The three techniques were rated by subjects on "preference" with 1 -to-7 scale $(1=$ lowest preference, and $7=$ highest preference). There was a significant difference between selection techniques in subjective preference, $\mathrm{F}(2,33)=31.2, \mathrm{p}<.0001$. Subjects gave ZWPS $($ mean $=6.61)$ a significantly higher rating than Area Cursor $($ mean $=3.13)$ and Take Off (mean $=4.25)$. Take Off was more preferred than Area Cursor. Subjects preferred ZWPS because ZWPS enables the user to easily select targets by zooming them, especially for very small (pixel level) targets with little void space.

\section{Experiment 2: Pressure Scroll}

The purpose of Experiment 2 was to examine whether pressure, as an additional control parameter, delivers benefits to stroke-based scrolling techniques. The stroke-based techniques with pressure control and without pressure control were experimentally compared. Although Scroll Bar is a standard technique in current GUIs our experiment did not involve this technique. The reason is that Scroll Bar is designed for mice and the result of our pilot studies showed that the stroke-based techniques without pressure control were better than Scroll Bar in pen-based interfaces.

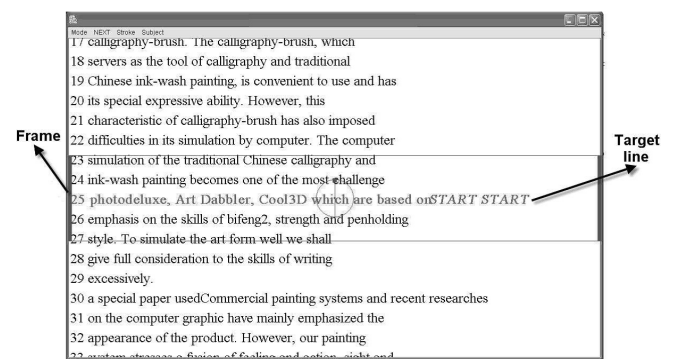

Fig. 8 The experimental interface for the reciprocal framing task.

\subsection{Participants}

Ten subjects (9 male, 1 female), all with previous experience with computers were tested for the experiment. The average age was 21.6. All subjects were right handed, and used the pen in the right hand.

\subsection{Apparatus}

The hardware used in Experiment 2 was the same to that in Experiment 1.

\subsection{Design and Procedure}

We conducted the experiment to compare stroke-based techniques with pressure to strokebased techniques without pressure. a vertical scrolling task was tested in Experiment 2. Closely following Hinckley, et al. ${ }^{9)}$ we evaluated our technique using a reciprocal framing task. In the vertical scrolling task, subjects scrolled down, then scrolled up, moving back and forth between two lines that are marked "START" and "END" respectively. We colored the "START" target line green and the "END" target line red. In Fig. $\mathbf{8}$ the red "frame" at the left of the experimental interface specified the width. For each target, the subjects scrolled until it entered the range of the screen identified by the frame. The frame was always centered on the screen. Once the target line was fully within the frame, the subject hit any key with 
the left hand. This selection key let the experimental interface know when the user judged the scrolling to be complete. If the target line had successfully fallen within the frame when the user struck the selection key, there was a short beep sound. If not, the user did not hear any sound, but we had instructed subjects to always continue to the next target (rather than trying to repair the error).

The design of the experiment crossed Method $\times$ scrolling Distance $(D) \times$ target Width $(W)$. We chose six representative distances: 48,72 , 96, 256, 416 and 570 lines, and one frame height: 3 lines. Each line is about 33 pixels (10 mm).

The participants read the instructions describing the experimental setup and the task. The experimenter then reviewed these instructions with the subjects and introduced the three methods. In order to familiarize them with the experimental environment, we set a practice session for each method. When the experimenter was satisfied that subjects had understood the task and could perform it correctly; the subjects completed the experiment unassisted. For each method, the subjects performed trials for each of the distance-width combinations in a random order. Each trial consisted of 7 phases of reciprocal movements between the target lines. The participants typically spent 40 to 50 minutes using the two methods.

\subsection{Results}

There is a line model ${ }^{1)}$ for movement time MT in scrolling:

$$
M T=a+b \cdot D
$$

where $a$ and $b$ are empirically determined constants, and $D$ is the distance (lines) between two target lines. We use this model to analyze the experimental results.

\subsubsection{Arc Stroke}

A repeated measure analysis of variance showed a significant main effect for the pressure means (hereafter referred to as Arcwith-pressure) and non-pressure means (hereafter referred to as Arc-without-pressure), $\mathrm{F}(1,119)=25.76, \mathrm{p}<.01$. There was a significant difference between the six distances, $\mathrm{F}(5,119)=43.11, \mathrm{p}<.001$. Separate paired samples t-tests were then run for each of the six distances, comparing the scrolling times for the pressure means and the non-pressure means.

For 256, 416 and 570 lines there were significant differences $(256: \mathrm{t}[9]=3.46, \mathrm{p}<.05 ; 416$ :

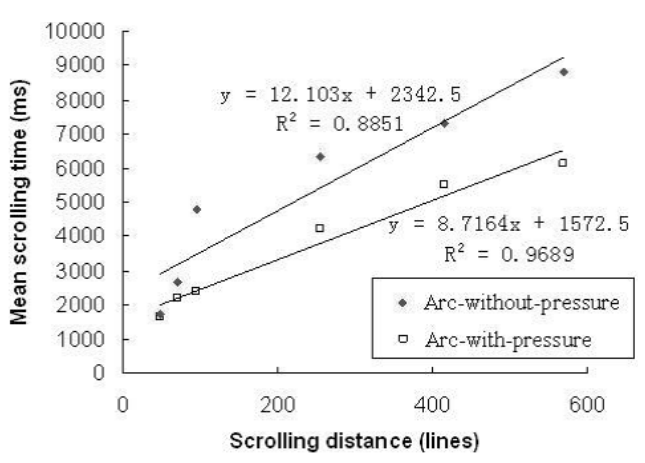

Fig. 9 Arc: mean movement time by scrolling distance for pressure mode and non-pressure mode.

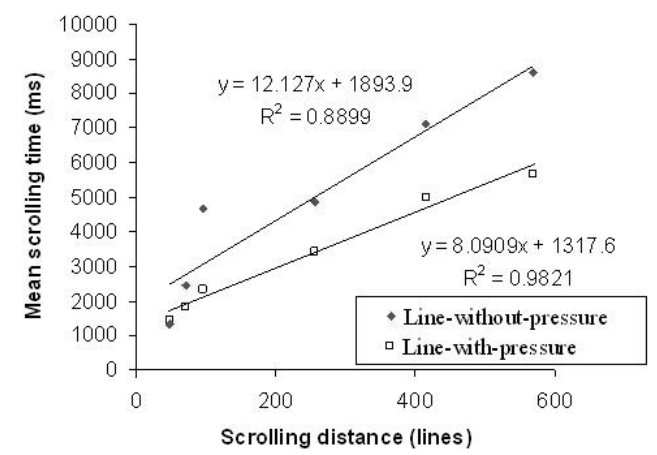

Fig. 10 Line: mean movement time by scrolling distance for pressure mode and non-pressure mode.

$\mathrm{t}[9]=2.66, \mathrm{p}<.05 ; 570: \mathrm{t}[9]=4.11, \mathrm{p}<.05)$. However, for 48, 72 and 96 there was no significant difference $(\mathrm{p}>.05)$ (see Fig. 9). For the pressure means, the error rates were $3.91 \%$ and for the non-pressure means, the error rates were $3.0 \%$. There was no significant difference between the two methods in error rate.

\subsubsection{Line Stroke}

A repeated measure analysis of variance showed a significant main effect for the pressure means (hereafter referred to as Linewith-pressure) and non-pressure means (hereafter referred to as Line-without-pressure), $\mathrm{F}(1,119)=18.11, \mathrm{p}<.01$. There was a significant difference between the six distances, $\mathrm{F}(5,119)=33.90, \mathrm{p}<.001$. Separate paired samples t-tests were then run for each of the six distances in order to compare the scrolling times for the pressure means and the non-pressure means.

For 96, 256, 416 and 570 lines, there were significant differences $(96: \mathrm{t}[9]=2.66 ; 256$ : $\mathrm{t}[9]=2.99, \mathrm{p}<.05 ; 416: \mathrm{t}[9]=3.17, \mathrm{p}<.05 ; 570$ : $\mathrm{t}[9]=3.96, \mathrm{p}<.05)$. However, for 48 and 72 there was no significant difference $(\mathrm{p}>.05)$ (see 
Fig. 10). The error rates were $3.71 \%$ for pressure means and $2.83 \%$ for non-pressure means. There was no significant difference between the two methods in error rate.

\subsubsection{Subjective Evaluation}

The methods were rated by the subjects on "preference" with 1-to-7 scale ( $1=$ lowest preference, and $7=$ highest preference). For both arc strokes and line strokes the analysis of the questionnaire showed no significant difference on subject preference between the pressure means and the non-pressure means.

\section{Discussion and Conclusions}

To explore the potentials of incorporating pressure into interactive techniques we designed two novel techniques ZWPS and Pressure Scroll to improve precise selections and scrolling performances in pen-based interfaces.

\subsection{ZWPS}

ZWPS utilizes pressure as a switch mode to couple two selection techniques (Point Cursor and a zoomable technique) to enable it convenient for both normal and precise selections. An experiment was conducted to compare it with Take Off and the improved Area Cursor. Experimental results indicate that ZWPS outperforms Take Off and the improved Area Cursor for small target selections. In particular, ZWPS still achieves good performances with small targets with little void space.

ZWPS provides a zoom function with enlarged target size to easily select very small targets, even with little void space around them. The user tends to zoom in small targets to reduce selection difficulties and visual burdens before selecting them by ZWPS. Moreover, ZWPS employs the selection manner of "Sliding into targets", which is significantly more accurate than "Directly landing on targets" ${ }^{21)}$. Although the improved Area Cursor has a big hot area, Area Cursor cannot take effect when selecting small targets which are close together. This is because, in such situations, the hot area of Area Cursor tends to cover more than one target and Area Cursor simply behaves as a Point Cursor. The selection manner of "Direct on" also tends to be quite inaccurate for such tasks. Take Off provides a cursor above a pentip with a fixed offset when touching the screen to achieve precise selection but it lacks a mechanism to reduce difficulties when selecting very small targets. The pressure activation mechanism enables ZWPS to flexibly and seamlessly switch between the zoom function and Point Cursor, which allows ZWPS to work like Point Cursor when selecting normal sized or big targets.

Pointing Lenses (Pressure-activated lenses) proposed by Ramos, et al. ${ }^{19)}$ are similar to ZWPS in their basic mechanism. However, their study only compared Pointing Lenses with three activation manners with Point Cursor by using selection tasks on a small single target. Comparison between Pointing Lenses and other promising cursors (like our experiment) was not conducted. Furthermore, we designed complex selection tasks in which the desired target is surrounded by distracters. Overall, we have proved the benefits of ZWPS through our experiment.

In GUIs some tasks require more than one step to be implemented with different operation methods. If a seamless switch mode is available, two or more techniques can be coupled together. ZWPS is such a hybrid technique that adapts to different types of tasks. Good selection performances achieved by ZWPS suggest that pressure should be a good alternative to switch modes.

\subsection{Pressure Scroll}

Pressure Scroll introduces pressure into the stroke-based scrolling techniques as an additional control parameter. The experimental results indicate that for both Arc stroke and Line stroke the pressure manners are better than the non-pressure ones. They also indicate that pressure is a beneficial control parameter in that it provides fine scale and large scale adjustments of the scrolling velocity.

Pressure reflects the information of the user's force imposed on pen-tips when using a pen to interact with screen. Therefore, the way of using pressure in a performance should not contradict the habits of imposing the force on the pen-tip. For an instance of scrolling performance: when fast scrolling a document, much heavier force is imposed on pen-tips. Pressure Scroll is based on this pre-existing behavior: when pressure is much heavier, the scrolling velocity becomes much larger.

\subsection{Implications for the Pressure- based UI Design}

The results of our experiments suggest several guidelines for the design of pen pressure techniques. First, our experiments have shown that pressure has the potential to advance interaction techniques. Second, the discrete or 
continuous manners of using pressure can be used as basic control methods for the designs of interaction techniques.

As shown by the experimental results, these two pressure-based techniques deliver some benefits to pen-based interactions. We can develop plug-ins to incorporate them into user interfaces. Some appropriate commands can be designed to allow switching between our proposed techniques and regular ones. For example, we can set the command buttons on the taskbar; when the user wants to perform operations by these new techniques, he/she would just click the command buttons to activate them. Or they can be activated by setting these commands as items in the pop-up menu, which is also convenient for the user.

Acknowledgments This study has been partially supported by Exploratory Software Project of IPA (Information-technology Promotion Agency in Japan), and Microsoft Research Asia Mobile Computing in Education Theme. We are grateful for the work and support of all the members of the Ren Lab in Kochi University of Technology. The authors also thank the anonymous reviewers for their valuable comments on an early version of this paper. Their contributions are gratefully acknowledged.

\section{References}

1) Anderson, T.: A Simple Movement Time Model for Scrolling, Proc. CHI2005, pp.11801183 (2005).

2) Albinsson, P.-A. and Zhai, S.: High Precision Touch Screen Interaction, Proc. CHI2003, pp.105-112 (2003).

3) Bandisch, P., Xie, X., Wang, C. and Ma, W.: Collapse-to-Zoom: Viewing Web Pages on Small Screen Devices by Interactively Removing Irrelevant Content, Proc. UIST2004, pp.9194 (2004).

4) Barrett, R., Olyha, J., Robert S. and Rutledge, J.: Graphical User Interface Cursor Positioning Device Having a Negative Inertia Transfer Function, Patent $\sharp 5,570,111$, IBM Corp (1996).

5) Buxton, W., Hill, R. and Rowley, P.: Issues and techniques in touch sensitive tablet input, Proc. ACM SIGGRAPH Conference, pp.215224 (1985).

6) Cockburn, A. and Savage, J.: Comparing Speed- Dependent Automatic Zooming with Traditional Scroll, Pan and Zoom Methods, Proc. of BCS HCI'03, pp.87-102 (2003).

7) Fitts, P.M.: The information capacity of the human motor system in controlling the amplitude of movement, Journal of Experimental Psychology, pp.381-391 (1954).

8) Herot, C. and Weinzapfel, G.: One-point touch input of vector information for computer displays, Proc. ACM SIGGRAPH Conference, pp.210-216 (1978).

9) Hinckley, K., Cutrell, E., Bathiche, S. and Muss, T.: Quantitative Analysis of Scrolling Techniques, Proc. CHI2002, pp.65-72 (2002).

10) Igarashi, T. and Hinckley, K.: Speeddependent automatic zooming for browsing large documents, Proc. UIST2000, pp.139-148 (2000).

11) Kurtenbach, G., Fitzmaurice, G., Baudel, T. and Buxton, B.: The Design of a GUI Paradigm based on Tablets, Two-hands, and Transparency, Proc. CHI'97, pp.35-42 (1997).

12) Kurtenbach, G., Sellen, A. and Buxton, W.: An empirical evaluation of some articulatory and cognitive aspects of 'marking menus', $\mathrm{Hu}$ man Computer Interaction, Vol.8, No.1, pp.123 (1993).

13) Li, Y., Hinckley, K., Guan, Z. and Landay, J.A.: Experimental Analysis of Mode Switching Techniques in Pen-based User Interfaces, Proc. CHI2005, pp.461-470 (2005).

14) Mackay, B., Dearman, D., Inkpen, K. and Watters, C.: Walk 'n Scroll: A Comparison of Software-based Navigation Techniques for Different Levels of Mobility, Proc. MobileHCI'05, pp.183-190 (2005).

15) Moscovich, T. and Hughes, J.: Navigating Documents with the Virtual Scroll Ring, Proc. UIST2004, pp.57-60 (2000).

16) Potter, R.L., Weldon, L.J. and Shneiderman, B.: Improving the accuracy of touch screens: an experimental evaluation of three strategies, Proc. CHI'88, pp.27-32 (1988).

17) Ramos, G. and Balakrishnan, R.: Fluid interaction techniques for the control and annotation of digital video, Proc. UIST2003, pp.105114 (2003).

18) Ramos, G., Boulos, M. and Balakrishnan, R.: Pressure Widgets, Proc. CHI2004, pp.487-494 (2004).

19) Ramos, G., Cockburn, A., Balakrishnan, R. and Beaudoiun-Lafon, M.: Pointing lenses: facilitating stylus input through visual-and motor-space magnification, Proc. CHI 200\%, pp.757-766 (2007).

20) Ramos, G. and Balakrishnan, R.: Zliding: Fluid zooming and sliding for high precision parameter manipulation, Proc. UIST2005, pp.143-152 (2005).

21) Ren, X. and Moriya, S.: Improving selection performance on pen-based system: A study of 
pen-based interaction for selection tasks, $A C M$ Trans. Computer-Human Interaction, Vol.7, No.3, pp.384-416 (2000).

22) Sears, A. and Shneiderman, B.: High Precision Touchscreens: Design Strategies and Comparison with a Mouse, International Journal of Man-Machine Studies, Vol.43, No.4, pp.593613 (1991).

23) Shneiderman, B.: Touch screens now offer compelling uses, IEEE Software, Vol.8, No.2, pp.93-94 (1991).

24) Smith, G. and Schraefel, M.: The Radial Scroll Tool: Scrolling Support for Stylus-or Touchbased Document Navigation, Proc. UIST2004, pp.53-57 (2004).

25) Tan, H., Srinivasan, M., Eberman, B. and Chang, B.: Human factors for the design of force-reflecting haptic interfaces, Proc. ASME Winter Annual Meeting, pp.353-359 (1994).

26) Worden, A., Walker, N., Bharat, K. and Hudson, S.: Making computers easier for older adults to use: area cursors and sticky icons, Proc. CHI'97, pp.266-271 (1997).

27) Zhai, S.: Human performance in six degree-of freedom input control, Ph.D. Thesis, University of Toronto (1995).

(Received April 3, 2007)

(Accepted September 3, 2007)

(Released December 5, 2007)

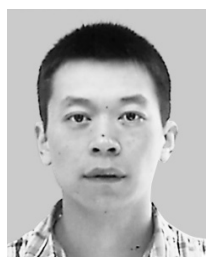

Jibin Yin is a Research Associate in the Department of Information Systems Engineering at Kochi University of Technology. He received the B.E. degree from Shenyang University of Technology, China, in 2000, the M.E. degree from Kunming University of Science and Technology, China, in 2003 and Ph.D. degree from Kochi University of Technology, Japan, in 2007, respectively. He is a member of the IEEE.

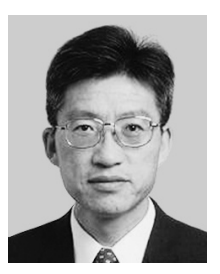

Xiangshi Ren is an Associate Professor in the Department of Information Systems Engineering at Kochi University of Technology. He received a B.E. degree in electrical and communication engineering, and M.E. and Ph.D. degrees in information and communication engineering from Tokyo Denki University, Japan, in 1991, 1993, and 1996, respectively. He was an instructor in the Department of Information and Communication Engineering at Tokyo Denki University from 1996 to 1999. After working for Tokyo Denki University, he has been at Kochi University of Technology since 2000. His research interests include all aspects of human-computer interaction, in particular, multimodal interactions, and user interface design and evaluation. He is a senior member of the IEEE, a member of the ACM, the IPSJ, the IEICE, and the Human Interface Society. 